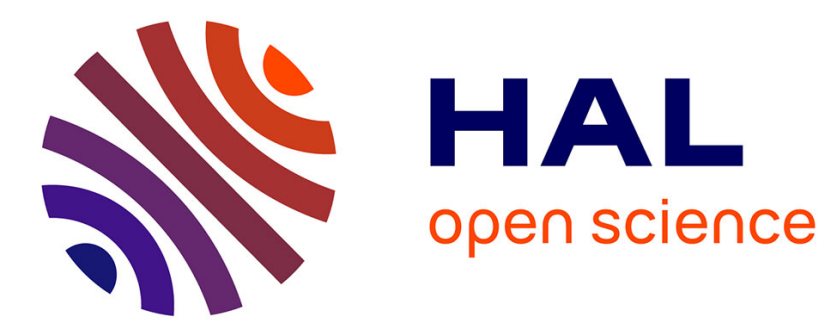

\title{
Implementation of a damage evolution law for dual-phase steels in Gurson-type models
}

Tudor Balan, Xavier Lemoine, Eric Maire, Anne-Marie Habraken

\section{To cite this version:}

Tudor Balan, Xavier Lemoine, Eric Maire, Anne-Marie Habraken. Implementation of a damage evolution law for dual-phase steels in Gurson-type models. Materials \& Design, 2015, 88, pp.1213-1222. 10.1016/j.matdes.2015.09.075 . hal-01273675

\section{HAL Id: hal-01273675 \\ https://hal.science/hal-01273675}

Submitted on 12 Feb 2016

HAL is a multi-disciplinary open access archive for the deposit and dissemination of scientific research documents, whether they are published or not. The documents may come from teaching and research institutions in France or abroad, or from public or private research centers.
L'archive ouverte pluridisciplinaire HAL, est destinée au dépôt et à la diffusion de documents scientifiques de niveau recherche, publiés ou non, émanant des établissements d'enseignement et de recherche français ou étrangers, des laboratoires publics ou privés. 


\title{
Implementation of a damage evolution law for dual-phase steels in Gurson-type models
}

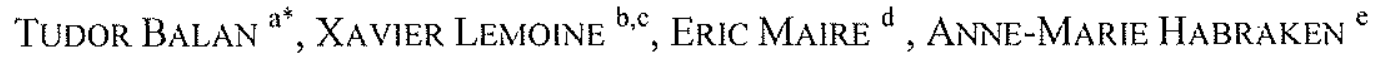

\footnotetext{
${ }^{a}$ Arts et Métiers ParisTech - Metz, LCFC, 4 rue A. Fresnel, 57078 Metz cedex 03, France

b ArcelorMittal R\&D Global Maizières S.A., voie Romaine, 57238 Maizières-Lès-Metz, France

${ }^{\mathfrak{c}}$ Arts et Métiers ParisTech - Metz, LEM3, UMR CNRS 7239, 4 rue A. Fresnel, 57078 Metz cedex 03, France

${ }^{d}$ INSA de Lyon, MATEIS CNRS UMR5510, 7 Avenue Jean Capelle, 69621 Villeurbanne, France

${ }^{\mathrm{e}}$ University of Liège, Département ArGEnCo, Division $\mathrm{MS}^{2} \mathrm{~F}$, Chemin des Chevreuils 1 , 4000 Liège, Belgium
}

* Corresponding author. Tel : +33.3.87.37.54.60; Fax: +33.3.87.37.54.70; e-mail : tudor.balan@ensam.eu

\begin{abstract}
This paper is a contribution to the phenomenological modeling of damage evolution in DP steels in the framework of Gurson's approach. It is based on recent results of X-ray tomography in-situ tensile tests and subsequent one-dimensional metallurgical void nucleation models proposed in [C. Landron et al., Scripta Materialia 63 (2010) 973-976]. A macroscopic void nucleation law for DP steels is proposed, covering a wide range of stress triaxialities. The respective effects of nucleation, growth and coalescence are clearly separated. Validations with respect to experimental porosity measurements were performed for several monotonic loading cases and for two loading sequences involving large strains and strain-path changes.
\end{abstract}

Keywords: GTN model, phenomenological void nucleation law, DP steels, finite element simulation, damage evolution. 


\section{Introduction}

Dual phase (DP) steels are widely used in the automotive industry as a basis for lightweight alternatives to classical designs with mild and high-strength-low-alloyed steels. DP steels offer remarkably high strength levels, allowing for significant reductions in thickness. However, these steels exhibit a specific formability behavior, where the necking phenomenon classically observed with lower steel grades competes with more sudden occurrences of fracture. The fracture behavior of DP steels is linked to its specific, heterogeneous microstructure. Damage was shown to initiate by the fracture of martensite inclusions, and/or by decohesion at martensite/ferrite interfaces. Subsequent strong strain localization occurs in the ferrite grains under the combined influence of martensite and voids (Kadkhodapour et al., 2011; Abid et al., 2015). Distribution, size and shape of the martensite grains have a strong influence. In order to model these local phenomena, several authors performed RVE-based simulations of DP steels by modeling the real microstructures of selected samples (Sun et al, 2009). In this framework, Sirinakorn et al. (2014) used the GTN model to describe the behavior of each phase and predict failure. Matsuno et al. (2015) used a fracture surface model and a cohesive model to predict void initiation at the scale of phases by the two aforementioned mechanisms. The model parameters are identified by comparison to in-situ SEM tensile tests, and were further used to successfully predict, for example, strain localization in the ferrite phase.

In view of metal forming applications, many authors attempted to model the failure of DP steels by macroscopic, mean field approaches. The "continuum damage mechanics" approach has been used, e.g., by (Niazi et al., 2013) to model the damage of DP steels, including damage anisotropy. However, given the microstructural specificity of DP steels, most authors focused on the "micromechanical damage approach" to predict the damage behavior of DP steels (Uthaisangsuk et al., 2011; Thuillier et al., 2012; Achouri et al., 2013). Void nucleation, growth and coalescence laws are continuously improved to extend their range of application to arbitrary stress states (Nahshon et al., 2008; Nielsen and Tvergaard, 2010; Cazacu et al., 2013), especially during shear, compression and bending-dominated forming processes. In particular, the classical Chu and Needleman (1980) equation was shown insufficient to describe the void nucleation phenomenon in DP steels. Based on in-situ measurements of void size and number evolutions, specific analytical equations were proposed for void evolution (Helbert et al., 1998; Maire et al., 2008; Saeidi et al., 2014) depending on plastic strain and explicitly incorporating stress triaxiality and some interface stress etc. Such formulations, specific to tensile loading, were generalized by Fansi et al. (2013) to arbitrary loading modes. Cao et al. (2014) used the nucleation law proposed by (Maire et al., 2010) with an additional shear term (Xue, 2008), while Malcher et al. (2014) used such an additional shear term with the classical Chu-Needleman nucleation law. Recent micro-tomography techniques allowed for the separation of the effects of void growth and void nucleation within the same experiment (Requena et al., 2014). This analysis revealed that stress triaxiality has an influence on the void nucleation phenomenon, and that this influence is similar to the one exhibited by void growth.

Traditional parameter identification for GTN-type models is based on the least-squares fitting of macroscopic force-displacement curves (Mahnken, 1999; Springman and Kuna, 2005), using tensile experiments on smooth or notched samples. This approach proved successful overall, but it does not allow for a fine discrimination of the void nucleation parameters. More 
information can be included in the identification by adding strain field measurements by image correlation (Abassi et al., 2013). More recently, these macroscopic measurements were combined with continuous in-situ SEM or X-ray diffraction micro-tomography measurement of void volume fraction, for refined identification of damage-related parameters (Ben Bettaieb et al., 2011; Cao et al, 2014). Ultimately, the GTN-like models are aimed to predict failure in forming processes. For this purpose, most authors simulate the actual process with refined finite element mesh. This procedure was used to predict forming limit diagrams (FLD) by simulating several Nakajima-type experiments (He et al., 2011; Kami et al., 2015). Mansouri et al (2014) used GTN with a bifurcation analysis to predict the FLDs of several sheet metals, including DP steel. At this macroscopic scale, DP steels are treated like most other metallic alloys.

The current study follows the previous efforts of Maire et al. (2008), Landron et al. (2010, 2011), Ben Bettaieb et al. (2011, 2012), and Fansi et al. (2013) on the experimental characterization, modeling and finite element (FE) implementation of Gurson type damage models. It investigates physically-based damage evolution laws specific for DP steels. Section 2 reviews very briefly the GTN model along with a recent porosity evolution law proposed by Landron et al. (2010) based on experimental X-ray tomography observations in DP steels. In Section 3, a phenomenological damage evolution law is derived in the framework of Gurson's approach. This law is inspired from the aforementioned model and attempts to extend its range of applicability. The proposed law is further investigated and compared to experimental results in Section 4, at large strains and under non-proportional loading histories.

\section{Modeling framework}

In the so-called micromechanical damage approach, a yield function for porous rigid-plastic metallic materials was initially proposed by Gurson (1977), and further extended by Tvergaard and Needleman (1984), in the form

$$
\Phi=\left(\frac{\sigma_{e q}}{\sigma_{Y}}\right)^{2}+2 q_{1} f \cosh \left(\frac{3}{2} q_{2} \frac{\sigma_{m}}{\sigma_{Y}}\right)-1-q_{3} f^{2},
$$

where $\sigma_{e q}=\sqrt{\frac{3}{2} \boldsymbol{\sigma}^{\prime}: \boldsymbol{\sigma}^{\prime}}$ is the macroscopic von Mises equivalent stress, $\sigma_{m}=\frac{1}{3}$ tro and $\boldsymbol{\sigma}^{\prime}=\boldsymbol{\sigma}-\sigma_{m} \mathbf{I}$ designate the mean and deviatoric Cauchy stress, respectively. In equation (1), $f$ stands for the void volume fraction, or porosity, and $\sigma_{Y}$ designates the flow stress of the dense matrix. In extended Gurson type models, the flow stress is a function of the cumulated plastic strain in the dense matrix $\bar{\varepsilon}$, as for example Swift's hardening function

$$
\sigma_{Y}=k\left(\varepsilon_{0}+\bar{\varepsilon}\right)^{n}
$$

where $k, n$ and $\varepsilon_{0}$ are material parameters. The normality rule defines the plastic strain rate

$$
\dot{\varepsilon}=\dot{\lambda} \frac{d \Phi}{d \sigma}
$$


and the following work equivalence principle is used to derive the relationship between macroscopic plastic work, equivalent stress and plastic strain rate in the dense matrix:

$$
(1-f) \sigma_{Y} \dot{\bar{\varepsilon}}=\boldsymbol{\sigma}: \dot{\varepsilon}
$$

For the purpose of this paper, simplified modeling is adopted in the framework of von Mises rigid-plasticity with isotropic hardening. For finite element applications, however, the proposed approach can be extended to anisotropic elasto-plasticity and kinematic hardening, following for example Ben Bettaieb et al. (2011, 2012), Shinohara et al. (2012) etc.

\subsection{Available models for the void volume fraction evolution}

To complete the model, an evolution law for the porosity $f$ has to be defined. The plastic incompressibility of the dense matrix leads to the classical evolution equation describing the growth of the existing voids:

$$
\dot{f}_{g}=(1-f) \operatorname{tr} \dot{\varepsilon}
$$

The nucleation of new voids during the loading history has been described in a phenomenological manner by Chu and Needleman (1980) with the following equation:

$$
\dot{f}_{n}=A_{C N}(\bar{\varepsilon}) \dot{\bar{\varepsilon}} \quad ; \quad A_{C N}=\frac{f_{C N}}{s_{C N} \sqrt{2 \pi}} \exp \left[-\frac{1}{2}\left(\frac{\bar{\varepsilon}-\varepsilon_{C N}}{s_{C N}}\right)^{2}\right]
$$

where $f_{C N}, s_{C N}$ and $\varepsilon_{C N}$ are material parameters. The mathematical form of this law implies that the nucleation phenomenon reaches maximum intensity at a particular value of cumulated plastic strain, and then diminishes and eventually vanishes as the strain continues to increase.

This nucleation law has been recently applied to the failure prediction of DP steels during forming, with good results (Thuillier et al., 2012; Troufflard et al., 2013; Sirinahorn et al., 2014). Mansouri et al. (2014) underlined that the porosity evolution law has first-order influence on strain localization; Hassanejadsl et al. (2013) found that for some forming processes this classical porosity evolution law was not able to predict failure with sufficient accuracy. Indeed, recent experimental investigations have revealed that the intensity of the nucleation phenomenon increases monotonically in DP steels, and does not reach a maximum as assumed by Eq. (6). Landron et al. (2010) monitored by X-ray tomography the number $N$ of voids per unit volume and the mean radius $R$ of the voids (supposed spherical), during insitu tensile tests on smooth and notched tensile specimens. To describe the increase in the number of voids per unit volume during loading, they proposed a void densily evolution law for DP steels where the main nucleation mechanism is decohesion at the interface between the martensite particles and the ferritic matrix. This law takes the form:

$$
\dot{N}=B \frac{\sigma}{\sigma_{c}}(1+\theta T)\left(1+\frac{N}{N_{0}}\right) \dot{\varepsilon}_{e q}
$$


where $B, \sigma_{C}$ and $N_{0}$ are material parameters, while $\theta$ is a factor allowing to calculate the local stress triaxiality $T$ in the vicinity of the interface between ferrite and martensite. In the framework of uniaxial tensile tests, Helbert et al. (1998) proposed to calculate $\theta$ as $\theta=\sigma /(\sigma-X)$, where $\sigma$ is the macroscopic uniaxial tensile stress and $X$ is the corresponding uniaxial backstress. In view of the application to three-dimensional simulations, this definition can be extended (Fansi et al., 2013) as $\theta=\sigma_{e q} / \tilde{\sigma}_{e q}$, where $\tilde{\sigma}_{e q}=\sqrt{\frac{3}{2}\left(\boldsymbol{\sigma}^{\prime}-\mathbf{X}^{\prime}\right):\left(\boldsymbol{\sigma}^{\prime}-\mathbf{X}^{\prime}\right)}$ is the von Mises equivalent value of the Cauchy stress tensor shifted by the backstress tensor $\mathbf{X}^{1}$.

This model has shown a good ability to describe the evolution of the void density in DP steels during tensile tests with different geometries and notches. Here, porosity is thus defined by means of the numerical density of voids $N$, and their average radius $R$. The void volume fraction $f$ is completely defined by these two quantities through the relationship

$$
f=\frac{V_{\text {voids }}}{V_{\text {total }}}=\frac{4 \pi}{3} N R^{3}
$$

and its evolution with time can be formally determined from the evolution rates of $N$ and $R$. The experimental measurements revealed that the mean void radius $R$ remains almost constant during loading - for various sample geometries and DP steel grades (Landron et al., 2011). This observation results from the competition between the growth of existing voids and the nucleation of new, smaller ones. In the particular case of a constant mean radius $R_{0}$, porosity and its evolution rate are defined by the simplified equations

$$
f=\frac{4 \pi}{3} N R_{0}^{3} \quad ; \quad \dot{f}=\frac{4 \pi}{3} R_{0}^{3} \dot{N}
$$

Combining equation (7) with Eq. (9) leads to the following evolution law for porosity:

$$
\dot{f}=B^{\prime}\left(\sigma_{e q}+\theta \sigma_{m}\right)\left(f_{0}+f\right) \dot{\varepsilon}_{e q}
$$

with $B^{\prime}=B /\left(N_{0} \sigma_{c}\right)$ and $f_{0}=\frac{4}{3} \pi N_{0} R_{0}^{3}$. Note that in this approach, no distinction is made between the contributions of nucleation $\left(\dot{f}_{n}\right)$ and growth $\left(\dot{f}_{g}\right)$. The macroscopic equivalent strain is used, while Eq. (6) makes use of the effective strain in the matrix. Finally, the stress triaxiality enters explicitly Eq. (10), while in Eq. (5) the trace of the plastic strain rate tensor was used instead.

\subsection{Predictions of porosity evolution under typical monotonic strain paths}

Chu and Needleman's (1980) empirical law Eq. (6) along with the void growth law Eq. (5) have been extensively applied in finite element simulations over the last decades. In contrast, Eq. (10) was only recently implemented in a FE code (Fansi et al., 2013), and it has been mainly validated for triaxiality values ranging between $1 / 3$ and 2/3 (Maire et al., 2008;

\footnotetext{
'In fact, Hill's 1948 anisotropic yield function was also used in (Fansi et al, 2013), for a more general case.
} 
Landron et al., 2010; Fansi et al., 2013). In view of its application for arbitrary loading modes, for example in metal forming processes, this law was tested in the current investigation under several monotonic loading modes. For this purpose, a rigid-plastic GTN model was developed based on equations (1)-(4), as described in the Appendix. Stress-driven radial loading conditions were considered, where the stress tensor was described as

$$
\sigma=\sigma^{*} \mathrm{~A}
$$

using an imposed stress mode tensor $\mathbf{A}$ and a driving scalar stress $\sigma^{*}$. The definition and physical significance of these quantities are given in Table 1 for the particular cases simulated within the current study. The table shows that several negative and positive triaxiality values were investigated in this way, ranging from $-\infty$ to $+\infty$. The used material parameters are summarized in Table 2 (Fansi et al., 2013). The parameters for the damage evolution law were identified by Landron et al. (2010) for a DP steel with $11 \%$ martensite. The parameters of the hardening law were identified with respect to the tensile stress-strain curve of the material in the rolling direction. In Figure 1, the model predictions are compared to the experimental data that served for the identification.

The predictions of the GTN model are shown in Figure 2 for the porosity evolution law (10). As expected, this law describes the influence of triaxiality very well when it takes moderate, positive values. In the case of simple shear and compression, a slight increase in porosity is predicted, while classical GTN models (using Eqs. (5)-(6) instead) would predict constant porosity for simple shear and decreasing porosity for compression. From an experimental viewpoint, the decrease of the void volume fraction can be inhibited partially or totally by the presence of particles inside the voids; experimental justification for an increasing porosity in these circumstances is however more questionable. Furthermore, unrealistic predictions of constant porosity are obtained for purely hydrostatic tension or compression. Similar behavior was observed with other analytical void nucleation expressions specific for DP steels, recently proposed in literature (Maire et al., 2008; Saeidi et al., 2014). All of these laws show a refined description of the stress triaxiality influence for moderately low, positive values, while exhibiting null or infinite porosity evolution rates for hydrostatic loading modes. The main purpose of this paper is to propose a corrective improvement of this family of models, without loss of its accuracy in tensile loading. 
Table 1. Definition of the stress-driven mechanical tests considered for simulation in the current investigation.

\begin{tabular}{|c|c|c|c|c|c|c|c|c|}
\hline & $\begin{array}{l}\text { Hydrostatic } \\
\text { compression }\end{array}$ & $\begin{array}{l}\text { Uniaxial } \\
\text { ompression }\end{array}$ & Rolling & $\begin{array}{l}\text { Simple } \\
\text { shear }\end{array}$ & $\begin{array}{l}\text { Plane } \\
\text { strain } \\
\text { tension }\end{array}$ & $\begin{array}{l}\text { Uniaxial } \\
\text { tension }\end{array}$ & $\begin{array}{l}\text { Axisym- } \\
\text { metric } \\
\text { tension }\end{array}$ & $\begin{array}{l}\text { Hydrostatic } \\
\text { tension }\end{array}$ \\
\hline$\sigma^{*}$ & $\left|\sigma_{11}\right|$ & $\left|\sigma_{11}\right|$ & $\left|\sigma_{33}\right|$ & $\sigma_{12}$ & $\sigma_{11}$ & $\sigma_{11}$ & $\sigma_{11}$ & $\sigma_{11}$ \\
\hline A & {$\left[\begin{array}{ccc}-1 & 0 & 0 \\
0 & -1 & 0 \\
0 & 0 & -1\end{array}\right]$} & {$\left[\begin{array}{ccc}-1 & 0 & 0 \\
0 & 0 & 0 \\
0 & 0 & 0\end{array}\right]$} & {$\left[\begin{array}{ccc}0 & 0 & 0 \\
0 & -1 / 2 & 0 \\
0 & 0 & -1\end{array}\right]$} & {$\left[\begin{array}{lll}0 & 1 & 0 \\
1 & 0 & 0 \\
0 & 0 & 0\end{array}\right]$} & {$\left[\begin{array}{ccc}1 & 0 & 0 \\
0 & 1 / 2 & 0 \\
0 & 0 & 0\end{array}\right]$} & {$\left[\begin{array}{lll}1 & 0 & 0 \\
0 & 0 & 0 \\
0 & 0 & 0\end{array}\right]$} & {$\left[\begin{array}{ccc}1 & 0 & 0 \\
0 & \gamma^{*} & 0 \\
0 & 0 & \gamma\end{array}\right]$} & {$\left[\begin{array}{lll}1 & 0 & 0 \\
0 & 1 & 0 \\
0 & 0 & 1\end{array}\right]$} \\
\hline$\sigma_{m}$ & $-\left|\sigma_{11}\right|$ & $-\frac{1}{3}\left|\sigma_{11}\right|$ & $-\frac{1}{2}\left|\sigma_{33}\right|$ & 0 & $\frac{1}{2} \sigma_{11}$ & $\frac{1}{3} \sigma_{11}$ & $\frac{T}{T+2 / 3} \sigma_{11}$ & $\sigma_{11}$ \\
\hline$\sigma_{e q}$ & 0 & $\left|\sigma_{11}\right|$ & $-\frac{\sqrt{3}}{2}\left|\sigma_{33}\right|$ & $\sqrt{3} \sigma_{12}$ & $\frac{\sqrt{3}}{2} \sigma_{11}$ & $\sigma_{11}$ & $\frac{1}{T+2 / 3} \sigma_{11}$ & 0 \\
\hline$T$ & $-\infty$ & $-\frac{1}{3}$ & $-\frac{1}{\sqrt{3}}$ & 0 & $\frac{1}{\sqrt{3}}$ & $\frac{1}{3}$ & $T$ & $\infty$ \\
\hline
\end{tabular}

$* \gamma=\frac{T-1 / 3}{T+2 / 3}$

Table 2. Parameters of the GTN model using the metallurgical porosity evolution law and the Swift hardening equation.

\begin{tabular}{ccccccccc}
\hline$f_{0}$ & $q_{1}$ & $q_{2}$ & $q_{3}$ & $B^{\prime}\left[\mathrm{MPa}^{-1}\right]$ & $\theta$ & $\begin{array}{c}K \\
{[\mathrm{MPa}]}\end{array}$ & $\varepsilon_{0}$ & $n$ \\
\hline $2 \times 10^{-5}$ & 1.5 & 1 & 2.25 & $3.18 \times 10^{-3}$ & 2 & 995 & 0.002 & 0.18 \\
\hline
\end{tabular}


a

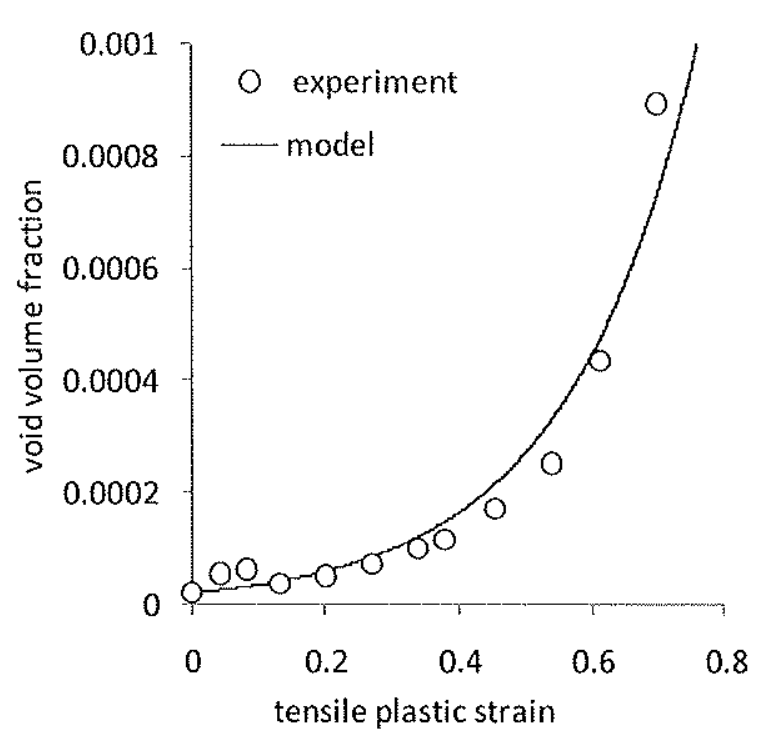

$\mathrm{b}$

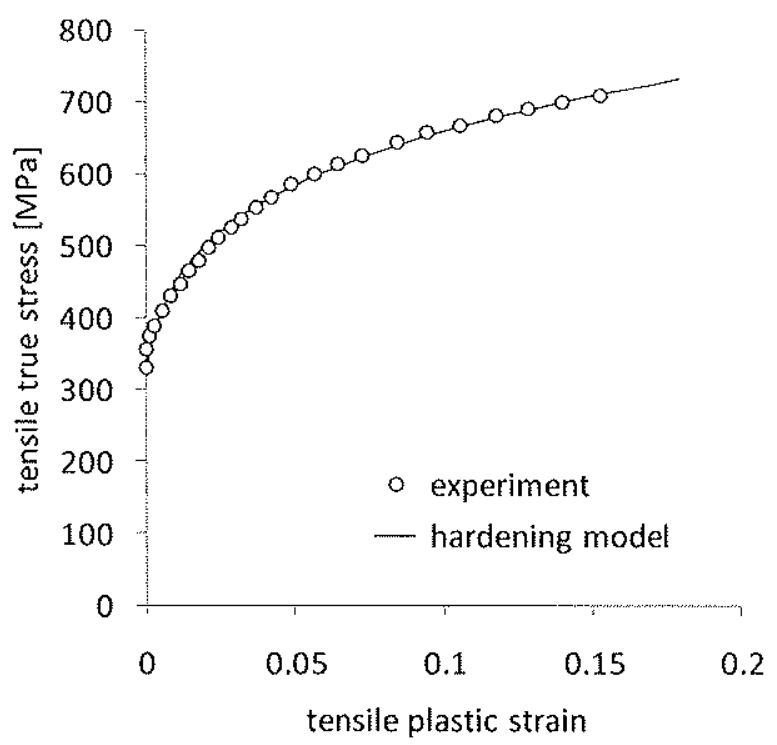

Figure 1. Comparison to uniaxial experiments of a) the void volume fraction predicted by Eq. $(10)$, and b) the hardening model. The void volume fraction evolution was measured during in-situ tensile tests on small-size specimens (Landron et al., 2010); the stress-strain curve was measured on a classic tension-compression machine using standard tensile samples (ISO 6892-1:2009).

a

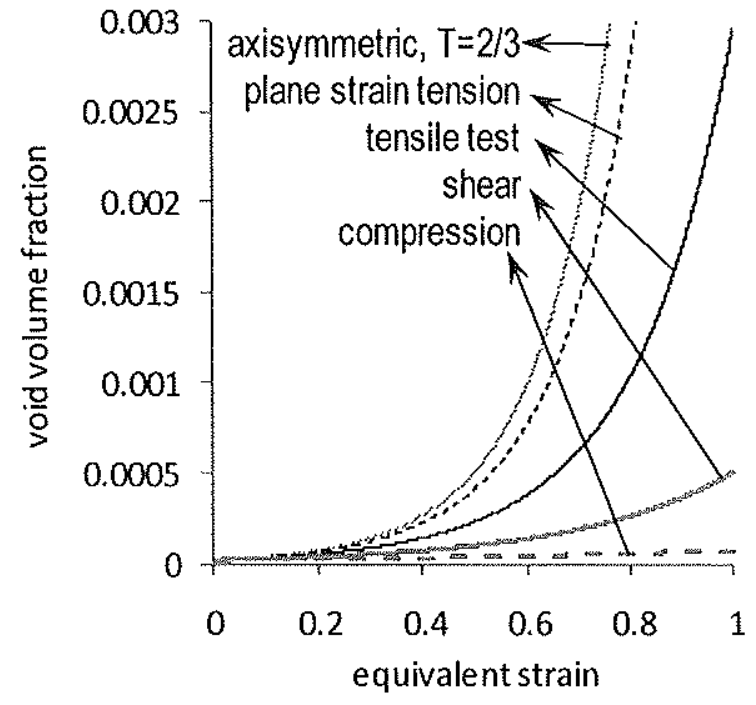

b

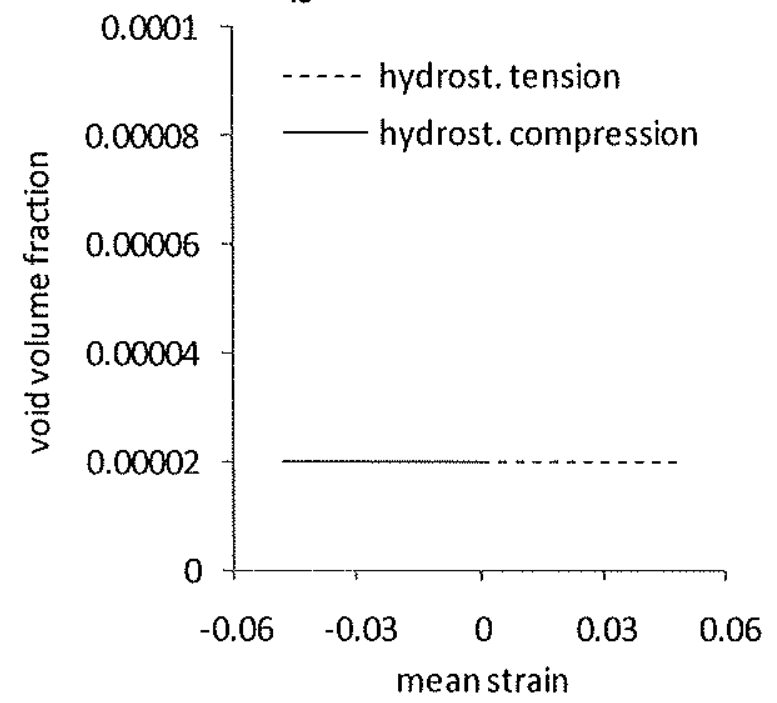

Figure 2. Predictions of void volume fraction evolutions using Eq. (10) for various triaxialities. In figure a), "axisymmetric, $\mathrm{T}=2 / 3$ " designates an axisymmetric tensile stress state where the stress components $\sigma_{22}=\sigma_{33}$ are determined in order to correspond to a stress triaxiality $T=2 / 3$. 


\section{Proposed model for void nucleation and growth in DP steels}

The metallurgically-based damage evolution law discussed in the previous section is the starting point of the current study. In view of FE implementation and application to arbitrary loading cases, its response under hydrostatic loading and negative triaxiality needs to be corrected, while its predictive response under low positive triaxiality should be preserved. This is the aim of the current section, where a phenomenological model is derived. Note that this pragmatic development is done in a phenomenological manner, based on experimental observations on DP steels. Consequently, the model may not be suitable for describing the damage evolution for other metallic materials (mild steels, aluminum alloys etc.).

As a first step, it is useful to recall that for loading histories involving either negative or infinite triaxialities, physically acceptable predictions can be obtained with the simple void growth law Eq. (5). Based on the plastic incompressibility of the matrix, this equation is classically used within GTN models. Its predictions are recalled in Figure 3. It must be noted that this void growth law is also triaxiality-sensitive, because it depends on the mean value of the plastic strain rate tensor.

Furthermore, the experimental monitoring of the dimensions of individual voids in DP steels have shown that their growth follows the trends of Rice and Tracey's (1969) classic law, which is of the same nature as the void growth law Eq. (5). Thus the predictions of the void growth law alone should represent a lower bound for the porosity evolution of this type of material, which is due to both void growth and nucleation. The most natural manner to ensure this effect is to make explicit the respective contributions of nucleation $\dot{f}_{n}$ and growth $\dot{f}_{g}$ in the porosity evolution law in the classic additive way:

$$
\dot{f}=\dot{f}_{n}+\dot{f}_{g}
$$

where $\dot{f}_{g}$ is assumed to be governed by Eq. $(5)^{2}$.

Next, the nucleation part of the evolution law must be determined in order to predict experimental observations. The expected properties of the nucleation law can be inferred from Figures 2 and 3. For the three tensile loading modes, the effect of triaxiality is the same in both figures, with the total porosity in Figure 2 being significantly higher than that due to growth (Figure 3). Thus it is obvious that the increase in porosity due to nucleation should follow the same trend. These figures also illustrate that the void growth law already provides the expected influence of stress triaxiality. Consequently, the porosity itself appears as a good candidate to convey this information to the nucleation law, avoiding the explicit use of the equivalent stress and strain. This is only a phenomenological speculation; no physical connection is assumed or claimed between the growth and nucleation mechanisms. In turn, it allows for a simple modeling approach with a reduced number of parameters. Indeed, a simple void nucleation law is suggested in the form

$$
\dot{f}_{n}=\alpha f \dot{\varepsilon}_{\text {eq }}
$$

\footnotetext{
${ }^{2}$ Different void growth laws have been proposed in literature to account for the particles that may be present inside the voids, their rotation, etc. For simplicity, Gurson's original void growth equation was selected in this study, but the approach is general.
} 